\title{
COINCIDENCIA ENTRE AGENDA MEDIÁTICA Y POLÍTICA EN LAS CUENTAS DE TWITTER DE LOS 4 CANDIDATOS CON MÁS VOTOS PARA LA ALCALDÍA DE QUITO
}

Coincidence between media and political agenda in Twitter accounts of the most voted 4 candidates running for Mayor in Quito

\author{
JORGE CRUZ \\ Pontificia Universidad Católica del Ecuador \\ jorgecruz.ec@gmail.com \\ ANDREA CARRILLO \\ Pontificia Universidad Católica del Ecuador \\ acarrillo745@puce.edu.ec \\ VANESSA ONATE \\ Pontificia Universidad Católica del Ecuador \\ vane12noboa@gmail.com
}

Resumen: Las intervenciones de los políticos en Twitter son medios con importancia para conocer cómo se complementa el discurso. La elección de alcalde de Quito 2019 presenta una oportunidad interesante para analizar cómo se construye ese pensamiento. Esta investigación encuentra cómo los tuits pueden reflejar la relación entre la agenda política y la agenda mediática. Estas dos agendas, se cruzan -en una perspectiva multimodal- a partir de cómo los políticos crean discurso (teorías de la propiedad y prominencia de las cuestiones) y cómo los medios lo hacen.

El estudio se refiere a los cuatro primeros candidatos en las elecciones seccionales (Yunda, Maldonado, Moncayo y Montúfar). El trabajo, a partir de una revisión a los planes de trabajo y a cientos de tuits de los candidatos, genera cuantificaciones y comparaciones en las temáticas trascendentales y comunes. Los hallazgos visualizan si se modificó o no el discurso político.

\begin{abstract}
The interventions of politicians on Twitter are important means to know how their discourse is complemented. Thus, the election of Mayor of Quito 20019 presents an interesting opportunity to analyze how that speech is built. This research finds how tweets can reflect the relationship between the political agenda and the media agenda. These two agendas intersect - in a multimodal perspective - based on how politicians create discourse (theories of Issue Ownership y Saliency) and how the media do it (Agenda Setting, Framing and Priming).

The study refers to the first four candidates in the sectional elections (Yunda, Maldonado, Moncayo and Montúfar). The paper, based on a review of the work plans and hundreds of tweets of the candidates, generates quantifications and comparisons in the transcendental and common themes. Findings visualize whether the political discourse was modified.
\end{abstract}

Palabras clave: Agenda Política, Agenda Mediática, Elecciones, Multimodal, Twitter Keywords: Political Agenda, Media Agenda, Elections, Multimodal, Twitter 


\section{INTRODUCCIÓN}

Las teorías políticas de la propiedad y de la prominencia de las cuestiones tienen un fuerte complemento de las teorías de la agenda mediática (priming, framing). Estas teorías, nacidas entre 1982 y 1996, se desarrollaron en un contexto de comunicación vertical, por lo que su aplicabilidad en nuevos escenarios sigue probándose en democracias consolidadas (Kleinnijenhuis, van Atteveldt and Dekkers, 2018; Kiousis, Strömbäck y McDevitt, 2015). El objetivo de la presente investigación fue trasladar y aplicar estas teorías en entornos digitales, donde se presume una horizontalidad de la información. Además, se plantea su aplicabilidad en un país cuya democracia está catalogada como "imperfecta" (Latinobarómetro, 2018). Así, se observó cómo la agenda digital de medios de comunicación afecta la agenda política y el comportamiento en Twitter de candidatos a la alcaldía de Quito, 2019.

En una primera sección, se hace una revisión de la literatura en torno al origen y evolución de la teoría de la propiedad y de la prominencia de las cuestiones, así como su relación con las agendas política y mediática. En una segunda sección, se describe el contexto en el que se desarrollaron las elecciones para la alcaldía de Quito 2019, esto con el fin de justificar la selección de una metodología mixta para la investigación: se desarrolló un análisis longitudinal de tipo cuantitativo y, posteriormente, a partir de técnicas mixtas, se observó la evolución de la postura en torno a las cuestiones de los candidatos más votados (Jorge Yunda, Luisa Maldonado, Paco Moncayo y César Montúfar). Vale la pena recalcar que los contenidos de entornos digitales fueron revisados desde una perspectiva multimodal. La investigación se plantea desde una hipótesis sobre una coincidencia entre las agendas políticas y mediáticas, es decir, que lo suscrito en planes de acción se reforzará en las ofertas y publicaciones de los candidatos.

\section{REVISIÓN TEÓRICA Y CONTEXTUAL}

\subsection{Elecciones, decisiones y cuestiones}

La competencia electoral en un sistema democrático consiste en enfatizar ciertas cuestiones y anular o silenciar otras. El criterio de selección, desde el punto de vista político, se puede determinar y estudiar a partir de dos teorías: (1) propiedad de las cuestiones y (2) prominencia de las cuestiones (Dolezal et al., 2014; Bélanger y Meguid, 2008).

En 1996, Petrocik postuló la teoría de issue ownership o Teoría de la propiedad de las cuestiones, que asociaba el dominio de un partido respecto a una política pública e intuía que este dominio no era más que una cuestión de percepción por parte de los votantes. En otras palabras, la teoría se basa en la reputación que mantiene un partido político respecto a cómo proceder con una política concreta. Así, desde esta perspectiva se presenta la pregunta: ¿qué partido político sería el mejor para lidiar con (cualquier cuestión política)? (Aragonés, Castanherira y Giani, 2012). Petrocick (1996) enfatiza el rol que mantienen las campañas para modificar el criterio de los votantes frente a la capacidad que tiene uno u otro partido. Advierte, además, que cada candidato pondrá un énfasis especial en la cuestión en la que sentirá poseer más ventaja o en la que ve debilidades entre sus oponentes. Así, en la medida en que se resaltan, enmarcan o anulan ciertos temas, esta teoría se relaciona con las de priming y framing. 
Walgrave, Lefevere y Tresch, en una revisión posterior de la teoría, distinguieron dos dimensiones para hablar de la propiedad: competencia y asociación. Los investigadores conceptualizan a la Asociación como: "la identificación espontánea entre temas y partidos específicos en la mente de los votantes" (Walgrave, Lefevere y Tresch, 2012, p. 771). Por otro lado, la dimensión Competencia es:

La reputación en políticas y programas, producida por una historia de atención, iniciativa e innovación hacia estos problemas, lo que lleva a los votantes a creer que uno de los partidos (...) es más sincero y está comprometido a hacer algo al respecto" (Petrocik en Walgrave, Lefevere y Tresch, 2012, p. 772).

En esta misma línea de ideas, Dolezal et al. (2014) advierten que en la actualidad los partidos políticos prefieren enfatizar selectivamente las cuestiones que les "pertenecen" en lugar de confrontarse directamente con los demás candidatos: "los partidos enfatizan desproporcionadamente los asuntos que "poseen" (2014, p. 57).

Sin embargo y si bien esta teoría tiene amplia aceptación como una de las bases para el estudio del comportamiento electoral, académicos subrayan la falta de consenso en torno al impacto que puede alcanzar (Walgrave, Tresch y Lefevere, 2015). De todos modos, dado que el objetivo de la presente publicación no se relaciona con la predicción del voto, esta singularidad no afecta los resultados.

Adicionalmente, dado que la Teoría de issue ownership toma en cuenta la percepción de los votantes, hay quienes advierten que esto también se relaciona con la prominencia de las cuestiones (Bélanger and Meguid 2008; Green y Jennings 2012; Lachat 2014; Walgrave et al., 2012). Así, aparece la Teoría de Issue saliency theory o prominencia de las Cuestiones; como punto de análisis en la competencia política en elecciones. La prominencia se relaciona con la pregunta: ¿Qué tan importante es una cierta cuestión para los votantes? Para ello, y en vista que los ciudadanos promedio son incapaces de entender completamente cada una de las políticas que debe implementar un gobierno, se crean macro-cuestiones que engloban varios aspectos que sean importantes para la sociedad (Budge, 1982).

Esto significará que los partidos políticos enfatizan un grupo común de cuestiones "más importantes" en cada campaña, pero que su credibilidad puede variar. Quiere también decir que la influencia que puede ejercer la pertenencia de una cuestión está condicionada por su prominencia (Budge y Farlie, 1983). Se intuyen prioridades de los votantes se hace, también, a partir del priming effect ${ }^{1}$, agenda setting ${ }^{2}$ y framing ${ }^{3}$, todas ellas aproximaciones del comportamiento mediático en la sociedad, una relación importante de revisar pues está establecido cómo los medios masivos de comunicación son capaces de persuadir e, incluso, 1. La idea del Priming se refiere se refiere al efecto de un evento y la manera en la cual se reacciona a algún estímulo posterior (Roskos-Ewoldsen, Roskos-Ewoldsen \& Carpentier 2002).

2. Jensen ofrece una definición sucinta sobre lo que es la Agenda Setting: "imposición de la información por los medios" (2014, p.256).

3. El concepto de Framing o Encuadre puede comprenderse como el proceso de selección de una sección de una realidad, a partir de cuatro variables: Presiones externas, Actitudes de la audiencia, Encuadres de los medios y Encuadres de las audiencias (Scheufele, 1999). 
de producir un cambio de actitud en los ciudadanos-votantes (Chaffee y Wilson, 1977, Gene Zucker, 1978; Lang y Lang, 1981).

\subsection{Agendas política y mediática}

La teoría del priming effect (Iyengar, Peters y Kinder, 1982) se considera una antecesora de la agenda setting; sostiene que los medios de comunicación son capaces de alterar la percepción humana a partir de la activación de preconcepciones. En otras palabras, una de las funciones de la memoria en la sociedad es la capacidad de completar capítulos futuros con base en las experiencias previas. La influencia de los medios -a través de la selección de palabras, actores y acontecimientos- logra manipular la memoria. Cuando se trata de política, en la que las personas carecen del conocimiento o entendimiento sobre asuntos políticos, estos basan sus decisiones en la información de los medios.

Queda establecido el papel de los medios como líderes de información en la actual sociedad, aunque su control total o compartición con los usuarios prosumidores es un tema de intenso y necesario debate. En este liderazgo, los medios definen de qué se habla (Agenda Setting), en sus propias plataformas y en las interacciones que se desprenden de su contenido, y son un aliado o un contrincante de los políticos que buscan alcanzar la bondad electoral. McCombs y Shaw (1972) sostienen que lo que sabemos del mundo lo conocemos a partir de lo que los medios nos presentan, ya que muchas de las cosas que se desencadenan y forman parte de asuntos políticos, están fuera de vista, de alcance y experiencia directa de los ciudadanos (McCombs y Shaw, 1972: 180).

La esencia y composición de agenda setting, se basa en el estudio de diferentes temas o actores sociales: agenda de medios o mediática, agenda pública y la agenda enfocada en nuestro estudio, agenda política. Todas buscan hablar de un tema central, una lista de asuntos enfocado en su área: cobertura mediática, pública y política. En esta última, enfocada en las acciones políticas, Budge y Farlie (1983) señalan que los partidos políticos suelen enfatizar un conjunto común de problemas importantes en una campaña electoral determinada, incluso si su credibilidad en esos temas varía, como se revisó antes en las teorías de Issue Ownership y Saliency.

Finalmente, es interesante ver cómo se integra el concepto de Encuadre o Framing. Los medios alteran lo que se conoce (como dice el Priming effect) y determinan los temas de los que se habla (Agenda Setting), pero es también importante no olvidar que esos temas tienen visiones particulares desde el cual las personas pueden conceptualizar particularmente un problema o reorientar su pensamiento sobre un problema desde una perspectiva particular. Así, los políticos intentan movilizar a los votantes para pensar desde las líneas políticas que se proponen desde los partidos, desde su relación a causa-efecto o los valores trascendentales (Chong y Druckman, 2017).

Por otro lado, es importante reconocer que los medios de comunicación definen sus contenidos a partir de ciertos elementos correspondientes a la naturaleza misma de su contenido y así construyen su agenda mediática, que "podría definirse como la lista de asuntos con cobertura mediática” (Zhu y Blood, 2002, pp. 100). Estas características se 
pueden agrupar bajo el concepto Noticiabilidad o incluso llamada teoría de las noticias. Esta perspectiva explica que los contenidos de los medios de comunicación dedicados a las noticias revisarán algunos detalles en lo que difundirán y a partir de una selección anterior dirán lo que es o no noticiable. Los criterios más relevantes que se pueden mencionar, a partir del trabajo de Ortells-Badenes (2014) son:

$\begin{array}{ll}\text { - } & \text { La novedad } \\ \text { - } & \text { El impacto y la trascendencia social } \\ \text { - } & \text { El conflicto } \\ \text { - } & \text { Ll interés humano } \\ \text { - } & \text { La proximidad } \\ \text { - } & \text { La dimensión del hecho } \\ \text { - } & \text { La rareza } \\ \text { - } & \text { La idea del progreso } \\ \text { - } & \text { La accesibilidad } \\ \text { - } & \text { La continuidad del hecho; } y, \\ \text { - } & \text { El equilibrio temático }\end{array}$

La autora también reconoce cinco parámetros que estarían fuera del control periodístico, pero que también influyen en cómo se construye la noticiabilidad y en un momento consecutivo, la agenda mediática: la observación de la competencia, el análisis de su público objetivo, la línea editorial, los factores económicos, y las limitaciones relacionadas con los recursos técnicos del medio (Ortells-Badenes, 2014: 209).

Antes de continuar con la explicación del objeto de estudio y la metodología a utilizar es necesario recapitular en la necesidad de interconectar las agendas políticas y mediáticas. Como se puede apreciar, en ambos casos tanto políticos como medios buscan afectar las decisiones de su público/electores. Para ello, el contenido de su discurso estará en relación con intereses presentes en la sociedad, que pueden obedecer o bien al desconocimiento, a las necesidades o a la forma en la que están construidos.

\subsection{Elecciones seccionales 2019}

La campaña electoral para alcanzar el curul de alcalde de Quito tuvo a 18 candidatos en la contienda ${ }^{4}$, que compitieron en un proceso electoral que arrancó el 5 de febrero y culminó el 22 de marzo de 2019. Durante esta breve temporada, los contendores realizaron encuentros con la comunidad, presentaciones en medios masivos, pautas en diversos medios, debates y gestionaron su presencia en entornos digitales.

4. Andrés Benavides, Xavier Buendía, María Sol Corral, Pablo Dávalos, Víctor Hugo Erazo, Patricio Guayaquil, Juan Carlos Holguín, Édgar Jácome, Luisa Maldonado, Paco Moncayo, César Montúfar, Andrés Pasquel, Carlos Sevilla, Juan Carlos Solines, José Vásquez, Paola Vintimilla, Jorge Yunda y Olivio Sarzosa. 
Tras la gestión del alcalde saliente, Mauricio Rodas, varios aspectos fueron determinantes sobre la labor que perseguiría el nuevo burgomaestre: transporte, basura, obras, corrupción, economía, seguridad, inclusión, parques (Plan V, 2019). Con estos temas en el aire, los candidatos generaron planes de trabajo y actividades comunicacionales para soportar las diferentes problemáticas de las que se embanderaron.

Al finalizar la campaña y las elecciones, los resultados; los cuatro primeros candidatos fueron Jorge Yunda, Luisa Maldonado, Paco Moncayo y César Montúfar. Los resultados, como se puede apreciar en la figura 1, le otorgaron la Alcaldía a Yunda (296 096 votos - 21,39\% de los votos); en el segundo lugar se ubicó Maldonado (255.007 - 18,42\%), seguida por Moncayo (246.142 - 17,78\%) y Montúfar (234.422 - 16.93\%). Es decir, entre los cuatro primeros lugares de los candidatos, tras las versiones oficiales (CNE, 2019), existió apenas un 4,46 de diferencia porcentual.

Figura 1. Resultados, primeros cuatro candidatos a la alcaldía de Quito

Votos válidos

\section{César Montúfar}

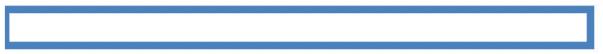

$16,93 \%$

Paco Moncayo

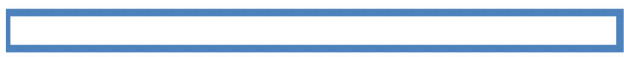

$17,78 \%$

Luisa Maldonado

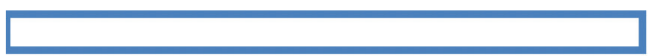

$18,42 \%$

Jorge Yunda

Fuente: Consejo Nacional Electoral, 2019. Elaboración: Los autores.

Todos los candidatos tuvieron una cuenta en Twitter activa y aunque las diferencias de uso de este canal digital fueron significativas, todos los políticos enviaron mensajes en la plataforma para fortalecer su discurso durante los 45 días de campaña. En un hallazgo interesante ${ }^{5}$, como se ve en la tabla 1, los dos candidatos que más interactuaron y publicaron en el servicio de microblogging también fueron los que más votos obtuvieron. Se verá después que los cuatro candidatos más votados encontraron en Twitter un entorno para discutir las temáticas que proyectaban su perfil como posible alcalde.

5El registro y análisis de los tuits de los candidatos a la Alcaldía de Quito forman parte del proyecto "Metodología de análisis multimodal de fenómenos políticos, sociales y noticiosos en contextos digitales" del Grupo de Investigación en Ciencias de la Comunicación (GicCom) de la Pontificia Universidad Católica del Ecuador. 
Tabla 1. Clasificación y número de tuits de candidatos.

\begin{tabular}{|c|c|c|}
\hline Candidato & Número de tuits & $\%$ de tuits \\
\hline Yunda & 1025 & $21,58 \%$ \\
\hline Maldonado & 588 & $12,38 \%$ \\
\hline Benavides & 441 & $9,28 \%$ \\
\hline Corral & 364 & $7,66 \%$ \\
\hline Holguín & 354 & $7,45 \%$ \\
\hline Moncayo & 283 & $5,96 \%$ \\
\hline Montúfar & 269 & $5,66 \%$ \\
\hline Vintimilla & 244 & $5,14 \%$ \\
\hline Jácome & 223 & $4,69 \%$ \\
\hline Buendía & 212 & $4,46 \%$ \\
\hline Pasquel & 161 & $3,39 \%$ \\
\hline Vásquez & 114 & $2,40 \%$ \\
\hline Solines & 113 & $2,38 \%$ \\
\hline Sevilla & 98 & $2,06 \%$ \\
\hline Zarzosa & 90 & $1,89 \%$ \\
\hline Guayaquil & 83 & $1,75 \%$ \\
\hline Erazo & 49 & $1,03 \%$ \\
\hline Dávalos & 39 & $0,82 \%$ \\
\hline & 4750 & $100,00 \%$ \\
\hline
\end{tabular}

Fuente: Elaborado por los autores.

\section{METODOLOGÍA}

Se plantea un análisis longitudinal de tipo cuantitativo en un inicio (para determinar la frecuencia de temas de las cuestiones políticas) y, a partir de estos resultados, se considera una aproximación mixta de análisis de discurso para observar la evolución de la postura de los candidatos más votados (Jorge Yunda, Luisa Maldonado, Paco Moncayo y César Montúfar).

Este análisis además tiene un génesis en la idea de revisar los contenidos de entornos digitales desde una perspectiva multimoda ${ }^{6}$, es decir desde una revisión integral y diversa de cómo se construyen y cómo se presentan estos contenidos. El análisis multimodal, de hecho, invita a reformar los conceptos tradicionales de cómo la gente se expresa, escribe o lee (Jewitt, 2005). En el caso de esta investigación, el análisis de los contenidos digitales se mirará a partir de la construcción del discurso político, afectado en primera instancia por las agendas políticas y a su vez por los hechos noticiosos presentes durante una determinada jornada o temporada, en este caso la lid electoral por la Alcaldía de Quito.

El análisis considera una comparación entre los proyectos políticos (manifiestos) de los candidatos a alcaldes y la evolución de su discurso en Twitter en tres cortes de la 6. Esto, también en concordancia con el proyecto de investigación antes mencionado. 
candidatura. La base de datos fue recolectada a través de Twitter Archiver, un complemento de GoogleSheets. Para determinar el posicionamiento de los candidatos en torno a las cuestiones, Marks (2007) propone que una de las aproximaciones es la codificación de los proyectos políticos. Este método es pertinente ya que refuerza la relación con las teorías de la Propiedad de las cuestiones y de la Prominencia de las cuestiones, tomando en cuenta estas definen que lo importante no es la posición "real" que muestran los candidatos, sino cómo esta información puede encontrarse y percibirse (Trechsel y Mair, 2011). Además, un análisis de los documentos y comunicaciones digitales (tuits), permite un análisis con mayor distancia, balance y transparencia de los datos.

En un primer momento, se clasificaron los planes políticos presentados por los candidatos en 17 temáticas; los datos se recolectaron a partir del archivo generado por diario El Comercio (2019).

\section{Tabla 2. Temas presentes en planes de campaña de los candidatos}

\begin{tabular}{|c|c|}
\hline Temas de campaña & \\
\hline Municipio & $\begin{array}{l}\text { Propuestas en torno a la forma en que se gestionará el municipio, } \\
\text { agilidad de trámites. Manejo de la comunicación y creación de marca } \\
\text { ciudad. }\end{array}$ \\
\hline Corrupción & $\begin{array}{l}\text { Rendición de cuentas y propuestas relacionadas con ética/moralidad. } \\
\text { Auditorías. Medidas de transparencia. }\end{array}$ \\
\hline Urbanismo/Territorialidad & $\begin{array}{l}\text { Propuestas sobre territorio y planificación urbanística. Promesa sobre } \\
\text { vivienda. Reordenamiento territorial. Legalización. Espacio público. } \\
\text { Recuperación de patrimonio. }\end{array}$ \\
\hline $\begin{array}{l}\text { Servicios Básicos Y } \\
\text { Saneamiento }\end{array}$ & Acceso a servicios básicos, recolección de basura. \\
\hline Turismo & $\begin{array}{l}\text { Promesas en torno a la potencialidad e impulso de la actividad } \\
\text { turística, conectividad internacional. }\end{array}$ \\
\hline Democracia Participativa & $\begin{array}{l}\text { Creación de mecanismos de participación de las decisiones del } \\
\text { Municipio. }\end{array}$ \\
\hline Educación & $\begin{array}{l}\text { Oferta de capacitaciones, creación de colegios/escuelas/universidades. } \\
\text { Promesas referentes a fomento de cultura. Acceso a tecnología y } \\
\text { conectividad. Cultura }\end{array}$ \\
\hline Inclusión Social & $\begin{array}{l}\text { Apoyo a emprendimientos, programas para discapacitados y grupos } \\
\text { vulnerables (incluidos migrantes). Regularización del trabajo e } \\
\text { inserción laboral. Seguro social. Vejez. }\end{array}$ \\
\hline Seguridad/Convivencia & $\begin{array}{l}\text { Programas para aumentar la presencia policial. Promesas referentes a } \\
\text { la delincuencia. Programas de prevención y gestión de riesgos. }\end{array}$ \\
\hline Movilidad & $\begin{array}{l}\text { Referente a parqueaderos, medidas pico y placa, bicicletas, transporte } \\
\text { público, cooperativas de taxis y buses. Vialidad. }\end{array}$ \\
\hline Deporte & $\begin{array}{l}\text { Actividades recreativas, infraestructura deportiva, ligas barriales, uso } \\
\text { del tiempo libre. }\end{array}$ \\
\hline Productividad & $\begin{array}{l}\text { Creación de cooperativas, promesas referentes al gasto público y a la } \\
\text { regulación de actividades económicas. Regulación de precios y } \\
\text { servicios. Producción agrícola y agropecuaria. Creación o protección a } \\
\text { los mercados. Emprendimiento. Desarrollo productivo. Canasta básica. } \\
\text { Empleo. }\end{array}$ \\
\hline Tributación & Promesas referentes a impuestos/ordenanzas. Sanciones. \\
\hline Ecología & $\begin{array}{l}\text { Control sobre la contaminación, limpieza de ríos/quebradas. Campañas } \\
\text { de reciclaje, ahorro de energía e incentivos (ecología). Programas a } \\
\text { favor de los animales. Paisaje urbano. }\end{array}$ \\
\hline Salud & $\begin{array}{l}\text { Programas contra la drogadicción, alcoholismo, etc. Creación de } \\
\text { centros de salud y fomento de planes de salud. Primeros auxilios. }\end{array}$ \\
\hline Campaña Electoral & Detalles y promoción de encuentros, eventos y cierres de campaña. \\
\hline Otros & $\begin{array}{l}\text { Tuits de información personal o que no entran en ninguna de las } \\
\text { categorías antes mencionadas. }\end{array}$ \\
\hline
\end{tabular}

Fuente: Elaborado por los autores. 
Una vez determinados los ejes temáticos, se procedió a clasificar los tuits de los candidatos $^{7}$ en estas mismas temáticas, existiendo la posibilidad de que un tuit se relacione con más de un tema a la vez. Dado el alcance de esta investigación, se procedió a seleccionar las propuestas y las cuentas de los cuatro candidatos más votados. Lo último se justifica con el hecho de que, a partir del quinto candidato, Juan Carlos Holguín $(6,86 \%)$, en adelante presentan valores porcentuales de votación muy bajos. Así, se procedió a un análisis cuantitativo de las temáticas más abordadas por los candidatos más votados y se determinó que son seis:

\section{Tabla 3. Temáticas de análisis y tuits}

\begin{tabular}{|c|c|}
\hline Temática & N. ${ }^{\circ}$ tuits totales \\
\hline Movilidad & 342 \\
\hline Seguridad y convivencia & 263 \\
\hline Inclusión social & 189 \\
\hline Corrupción & 242 \\
\hline Productividad & 182 \\
\hline Democracia participativa & 94 \\
\hline
\end{tabular}

Fuente: Elaborado por los autores.

En este punto, vale la pena mencionar que no es de sorprender que estas sean las temáticas tratadas con mayor intensidad puesto que seis de ellas se relacionan directamente con las grandes preocupaciones ciudadanas de acuerdo con Latinobarómetro (2018): distribución de la riqueza, confianza en las instituciones, violencia, corrupción y satisfacción con la vida. Por su parte, la temática de "Ecología, medio ambiente y fauna urbana" se relaciona con uno de los "grandes desafíos que enfrenta América Latina en el siglo XXI” (Centeno y Lajous, 2017). De los candidatos a la Alcaldía y sobre todo para los cuatro políticos parte de este análisis, Jorge Yunda fue el que más tuits publicó sobre esta temática, particularmente sobre la tenencia y rescate de animales. Yunda difundió 295 tuits al respecto (el 26,4\% de sus publicaciones, mientras que Paola Vintimilla fue segunda en este apartado con 20 tuits; esta diferencia marca que la temática no ingrese en el análisis comparativo.

Para el resto de las temáticas, tomando en consideración que las cuestiones políticas están determinadas por el auto posicionamiento de los partidos políticos (Treschel y Mair, 2011), se determinaron entre seis y dos afirmaciones por cada tema de acuerdo con su alcance. Estas afirmaciones fueron evaluadas mediante análisis de discurso en los 4 momentos antes mencionados.

7. Las cuentas fueron monitoreadas desde el inicio de la campaña electoral en 3 cortes de tiempo similar: del 5 al 19 de febrero, del 20 de febrero al 6 de marzo y del 7 al 21 de marzo. 
Tabla 4. Temas de la agenda de tres medios impresos

\begin{tabular}{|c|c|}
\hline Tema & Afirmaciones \\
\hline Movilidad & $\begin{array}{l}\text { - Se necesitan más parqueaderos públicos en Quito. } \\
\text { - La medida "pico y placa" debe ser endurecida (ampliada). } \\
\text { - Se deben ampliar los servicios de transporte público (rutas u } \\
\text { horarios). } \\
\text { - Se deben aumentar las unidades de transporte público. } \\
\text { - Se apoya a las movilidades no motorizadas. }\end{array}$ \\
\hline Seguridad y convivencia & $\begin{array}{l}\text { - Quito necesita planes para combatir la inseguridad. } \\
\text { - Se debe combatir la delincuencia. } \\
\text { - Se deben crear programas de prevención y gestión de riesgos. } \\
\text { - Quito debe disminuir la violencia de género. }\end{array}$ \\
\hline Inclusión social & $\begin{array}{l}\text { - Se debe incentivar el emprendimiento. } \\
\text { - Los programas para discapacitados y grupos vulnerables } \\
\text { (incluidos migrantes) son beneficiosos para los quiteños. } \\
\text { - El trabajo debe ser regularizado. } \\
\text { - Se debe procurar la inserción laboral. }\end{array}$ \\
\hline Corrupción & $\begin{array}{l}\text { - La alcaldía debe rendir cuentas de su gestión e inversiones. } \\
\text { - El alcalde y su equipo de trabajo deben mostrar ética y ser } \\
\text { morales. } \\
\text { - El Municipio debe ser sometido a auditorías. } \\
\text { - Se deben incorporar medidas de transparencia en los procesos del } \\
\text { municipio. }\end{array}$ \\
\hline Productividad & $\begin{array}{l}\text { - Se deben crear cooperativas para facilitar el acceso a servicios } \\
\text { bancarios. } \\
\text { - } \\
\text { - Se Municipio debe regular precios y servicios. } \\
\text { - El Municipio debe preocuparse por la creación y protección de } \\
\text { los mercados. }\end{array}$ \\
\hline Democracia participativa & $\begin{array}{l}\text { - Se deben crear mecanismos para que la gente participe en las } \\
\text { decisiones del municipio. } \\
\text { - Se deben fortalecer las organizaciones ciudadanas. }\end{array}$ \\
\hline
\end{tabular}

Fuente: Adaptación de Treschel y Mair, 2011.

Dichas aseveraciones son contestadas de manera estandarizada y convertidas a una cifra numérica de acuerdo con la siguiente lógica: 
- $\quad$ En completo desacuerdo

- En desacuerdo

- $\quad$ Postura neutral, no habla del tema

- De acuerdo

- $\quad$ En completo acuerdo Agenda mediática 12345

Para el análisis se buscó construir un contexto de la temporada electoral, a partir de la visión de tres medios impresos, sobre todo con sus redacciones y administraciones en Quito: El Comercio, La Hora y el vespertino Últimas Noticias ${ }^{8}$. Se realizaron monitoreos entre el 5 de febrero y el 23 de marzo del 2019 (día previo a las elecciones).

Este levantamiento de información buscó el reunir todos los temas en el que los candidatos podrían encontrar mayor relación y en los que podían aprovechar espacio en los medios; es decir, lograban crear noticia a partir de su discurso. A su vez, si bien existía previamente una propuesta política, se buscó la relación de ésta con la agenda noticiosa.

Tabla 5. Temas de la agenda de tres medios impresos

\begin{tabular}{|c|c|c|c|c|}
\hline \multicolumn{2}{|r|}{ Temáticas relevantes } & \multirow{2}{*}{$\begin{array}{c}\text { El Comercio } \\
\mathrm{x}\end{array}$} & \multirow{2}{*}{$\begin{array}{c}\text { La Hora } \\
\mathrm{x}\end{array}$} & \multirow{2}{*}{$\begin{array}{l}\text { Ultimas } \\
\text { Noticias }\end{array}$} \\
\hline 1 & $\begin{array}{l}\text { Servicios públicos: sistemas de } \\
\text { agua potable, alcantarillado, } \\
\text { recolección de basura. }\end{array}$ & & & \\
\hline 2 & $\begin{array}{l}\text { Movilidad: baches en las } \\
\text { calles, adoquinado. }\end{array}$ & $\mathrm{x}$ & $\mathrm{x}$ & \\
\hline 3 & Seguridad & $\mathrm{x}$ & $\mathrm{x}$ & $\mathrm{x}$ \\
\hline 4 & Fuentes de trabajo & $\mathrm{x}$ & $\mathrm{x}$ & \\
\hline 5 & No violencia & $\mathrm{x}$ & $\mathrm{x}$ & \\
\hline 6 & Participación ciudadana & $\mathbf{x}$ & $\mathrm{x}$ & \\
\hline 7 & Regularización de barrios & $\mathrm{x}$ & $\mathrm{x}$ & \\
\hline 8 & Corrupción & $\mathbf{x}$ & $\mathrm{x}$ & \\
\hline 9 & $\begin{array}{l}\text { Igualdad de discapacidades } \\
\text { y género }\end{array}$ & $\mathrm{x}$ & $\mathrm{x}$ & \\
\hline 10 & Red de ciclistas & & $\mathrm{x}$ & \\
\hline 11 & Eliminación de taxi rutas & & $\mathrm{x}$ & \\
\hline
\end{tabular}

Fuente: Elaborado por los autores

8. El Comercio es el sitio web más visitado de un medio impreso según el ranking de Alexa, La Hora se ubica en el cuarto lugar y Últimas Noticias en el décimo primero. 
Como quedó establecido, la primera parte del análisis consideró los planes de trabajo de los candidatos, a la luz de las temáticas más recurrentes en las publicaciones en Twitter; posteriormente se entabló una cuantificación de cada una de las propuestas. Estas calificaciones se replicaron en las publicaciones de cada candidato (a partir de la temática) y se cuantificaron de la misma manera. Finalmente, se construyeron correlaciones al restar el valor de la calificación del plan de campaña del valor de cada uno de los cortes (Tabla 6). Sobre este valor se revisará cómo las cuentas de Twitter evidencian mayor interés en hablar en ciertas temáticas (valor positivo) o un desinterés de la temática a partir de lo propuesto en campaña (valor negativo); mientras el valor esté en cero o cerca de ese valor, el candidato se habrá comportado -discursivamente- tal cual lo presentó en su plan de campaña.

Tabla 6. Coeficientes entre plan de trabajo y cortes por candidato

\begin{tabular}{|c|c|c|c|c|}
\hline Temática & Candidato & Feb 0519 & Feb 20 a Mar 06 & Mar 07 a 21 \\
\hline \multirow[t]{4}{*}{ Movilidad } & Yunda & 0,2 & $-0,4$ & $-0,2$ \\
\hline & Maldonado & $-0,4$ & 0,6 & 0 \\
\hline & Moncayo & 0,4 & 0,6 & 0,8 \\
\hline & Montúfar & $-1,2$ & $-0,8$ & $-0,8$ \\
\hline \multirow[t]{4}{*}{ Seguridad } & Yunda & $-1,5$ & $-0,5$ & $-0,5$ \\
\hline & Maldonado & $-0,5$ & $-0,5$ & $-0,25$ \\
\hline & Moncayo & 1 & 1 & 0,75 \\
\hline & Montúfar & $-1,5$ & -2 & $-1,75$ \\
\hline \multirow[t]{4}{*}{ Productividad } & Yunda & 0,25 & 0,25 & 0 \\
\hline & Maldonado & 0,25 & 0,75 & 0,25 \\
\hline & Moncayo & -1 & $-0,5$ & $-0,75$ \\
\hline & Montúfar & $-0,5$ & $-0,5$ & 0,25 \\
\hline \multirow[t]{4}{*}{ Corrupción } & Yunda & 0,5 & 0 & 0,25 \\
\hline & Maldonado & 0,5 & 1 & 0,5 \\
\hline & Moncayo & $-0,5$ & $-0,75$ & $-0,75$ \\
\hline & Montúfar & -1 & $-0,75$ & $-0,25$ \\
\hline \multirow[t]{4}{*}{ Democracia participativa } & Yunda & 0,5 & 1 & 1 \\
\hline & Maldonado & 0,5 & 1 & 0 \\
\hline & Moncayo & -1 & $-0,5$ & $-0,5$ \\
\hline & Montúfar & $-0,5$ & $-0,5$ & 0 \\
\hline \multirow[t]{4}{*}{ Inclusión social } & Yunda & -1 & $-0,75$ & $-0,75$ \\
\hline & Maldonado & 0 & 0,5 & 0,25 \\
\hline & Moncayo & $-0,5$ & -1 & $-1,25$ \\
\hline & Montúfar & $-1,5$ & -1 & -1 \\
\hline
\end{tabular}

Fuente: Elaborado por los autores 


\section{HALLAZGOS}

En la temática de Movilidad -referente a parqueaderos, medida Pico y Placa, bicicletas, transporte público, cooperativas de taxis y buses- aparece un interesante comportamiento (Figura 2) de las publicaciones del candidato Montúfar, que se distancia en cada uno de los cortes de su posición en el plan de campaña. Por otro lado, el candidato Moncayo se interesó en la temática de manera progresiva a partir de cómo la campaña y el tiempo avanzaba. Solamente este candidato tomó una postura "en completo acuerdo" respecto al apoyo de movilidades no motorizadas.

La movilidad también fue un aspecto recurrente en los medios a la par de todo el proceso informativo que se vivió en Twitter. Las noticias se relacionaron a la problemática de baches en las calles, daños en el adoquinado, inconformidad de la red de ciclistas y los taxi-rutas. Las noticias destacadas fueron:

1. Los grandes retos del nuevo Concejo de Quito (Publicada el 02/03/2019)

2. Planes de Gobierno de los aspirantes a la Alcaldía (28/02/2019)

3. 14 de 18 candidatos a alcalde de Quito asistieron a debate de propuestas (08/03/2019)

4. Las ofertas de los candidatos a la Alcaldía de Quito no incluyen plan presupuestario $(10 / 03 / 2019)$

En armonía con lo que Budge y Flaire (1983) advierten, este hallazgo demuestra cómo la agenda política puede variar de acuerdo con la prominencia de una cuestión. En otras palabras, dado que la movilidad, desde los medios de comunicación, fue catalogada como "un reto" (El Comercio, 2019), los candidatos toman diferentes posiciones a la descrita inicialmente.

\section{Figura 2. Resultados de temática: Movilidad}

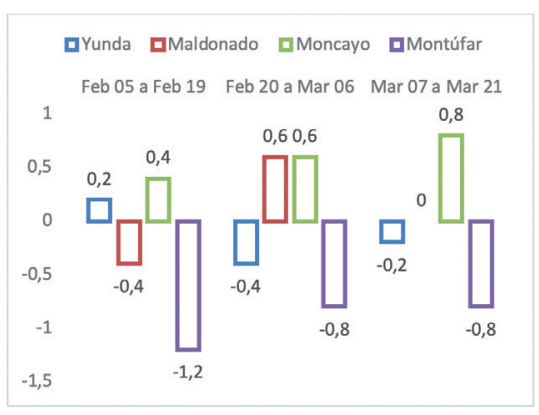

Fuente: Elaborado por los autores.

En seguridad, lo notable en la relación está en que, salvo el general Paco Moncayo, los candidatos no le prestaron atención discursiva en relación con su plan de campaña (Figura 3); particularmente César Montúfar abandonó el tema de manera sostenida en los tres cortes analizados. Moncayo, como se menciona, respondió a esta temática con mayores 
intervenciones al respecto. Por su lado, las noticias se refirieron al pedido de la población por el incremento de robos y asaltos a mano armada, las inquietudes de los ciudadanos sobre la inseguridad y la delincuencia.

En este punto, cabe la pena resaltar el completo silencio que mantuvieron los candidatos en relación con la gestión de riesgos (que fue solamente contemplada en los planes de gobierno), lo que demuestra la falta de cultura de prevención. Las noticias y entrevistas que abordaron varios puntos importantes sobre esta temática fueron las siguientes:

1. Los desafíos de los candidatos a alcalde (14/02/2019)

2. Luisa Maldonado: 'Convertiré al Municipio en un lugar de emprendedores' (27/02/2019)

3. Planes de Gobierno de los aspirantes a la Alcaldía (28/02/2019)

4. Jorge Yunda: 'Yo creo que el quiteño sale con mucha zozobra a la calle' (06/03/2019)

5. Las ofertas de los candidatos a la Alcaldía de Quito no incluyen plan presupuestario $(10 / 03 / 2019)$

6. Paco Moncayo: 'No seré empleador de nadie, no debo nada a nadie' (20/03/2019)

\section{Figura 3. Resultados de temática: Seguridad}

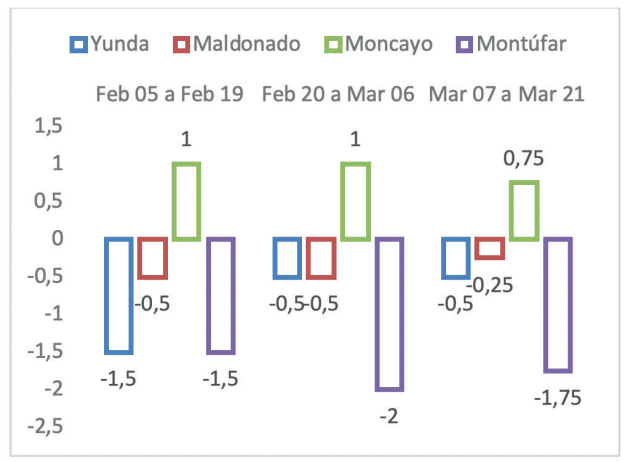

Fuente: Elaborado por los autores.

El tema de productividad no tuvo grandes picos para los candidatos. Es la temática con menor cantidad de aportes positivos sobre los coeficientes presentes a partir del plan de campaña Figura (4). Solo la candidata Luisa Maldonado tuvo publicaciones superiores en cada corte, respecto a lo que planteaba su plan de campaña. Yunda también tuvo un pequeño pico, pero menor al que presentaban otras temáticas.

Moncayo y Montúfar, por su parte, se refirieron al tema en menor medida de lo que se habría planteado el plan, particularmente Moncayo. Por su parte, los medios llevan su punto de atención a la regulación de barrios y la creación de nuevas ofertas de empleo. La noticia más evidente es: "Trabajos de época electoral son un paliativo para el desempleo" (27/02/2019). 
Figura 4. Resultados de temática: Productividad

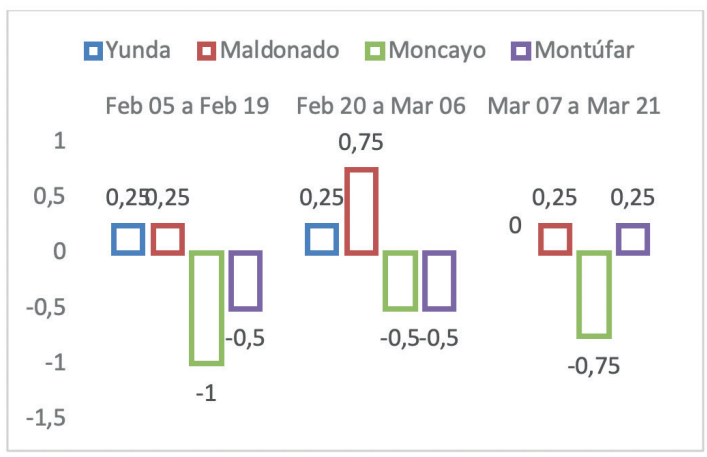

Fuente: Elaborado por los autores.

En la temática de corrupción lo interesante aparece en el comportamiento discursivo en los dos espectros (positivo y negativo). Por un lado, como se muestra (Figura 5) Yunda y especialmente Maldonado se refirieron a aspectos relacionados con la corrupción durante su campaña. Sin embargo, el discurso de la candidata no se centraba en sus promesas de transparencia, sino que fue una discursiva rival hacia Paco Moncayo y la petición de la fiscalización del proceso del Metro de Quito.

Lo más interesante está en la difusión de mensajes de César Montúfar que fue el candidato que más se refirió a tema en su plan de campaña. Durante los tres cortes, sus aportes se cuantificaron con un coeficiente inferior al de su plan, aunque es importante mencionar que inició con un -1 y terminó con un -0,25. En los medios, se toparon temas como la obligación de la alcaldía para rendir cuentas de su gestión e inversiones. Entre las noticias más importantes sobre esta temática, tenemos las siguientes:

1. Los desafíos de los candidatos a alcalde (14/02/2019)

2. Planes de Gobierno de los aspirantes a la Alcaldía (28/02/2019)

3. Las ofertas de los candidatos a la Alcaldía de Quito no incluyen plan presupuestario $(10 / 03 / 2019)$

4. Unas elecciones con varios contrasentidos (18/03/2019)

El cambio que se evidencia en César Montúfar, desde la teoría, se relaciona con la variabilidad que el candidato puede experimentar en el tema de "credibilidad" y “capacidad" (D'Ancona, 2016). Cada candidato decide la cuestión de la que se abanderará, pero esta selección se realiza en tono con lo que los votantes creen que será capaz de hacer el candidato. Así, tras el alto impacto que ha recibido el tema de "corrupción" — que resulta ser transversal en todo proceso de gobierno-, es natural que los candidatos se muevan a otras cuestiones. 
Figura 5. Resultados de temática: Corrupción

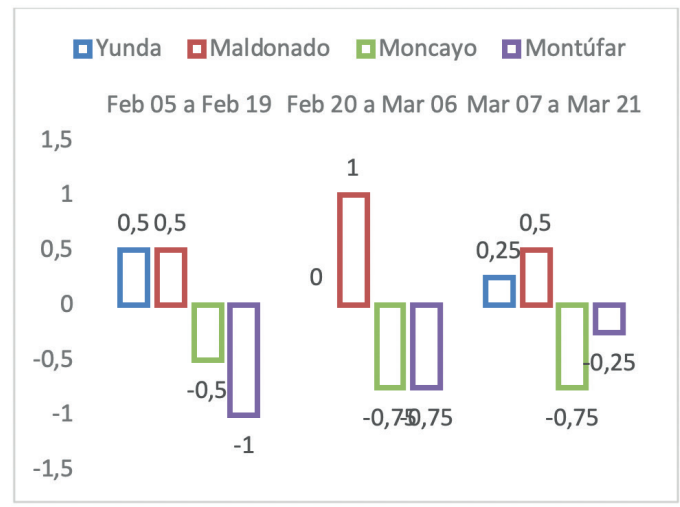

Fuente: Elaborado por los autores.

En el apartado de Democracia Participativa, lo interesante es mirar cómo los dos candidatos que al final obtuvieron más votos son los que tuvieron mayor énfasis en esta temática (Figura 6). Los otros dos candidatos no obtuvieron la misma cuantificación que lo que se presentó en sus planes de trabajo. Se debe dar especial énfasis al hecho de que, Democracia Participativa está relacionada con la creación y respeto de espacios de participación ciudadana en la toma de decisiones. De esto, con respecto a la creación y fortalecimiento de nuevos espacios de discusión, solo el candidato Montúfar expresa una fuerte posición a favor. Los demás candidatos relacionan la democracia participativa con la escucha en barrios y mercados.

Los medios refieren a la democracia participativa como una participación ciudadana donde todos estén informados para poder decidir y tener un voto consciente. Las noticias más importantes en esta temática fueron las siguientes:

1. Elecciones 2019: Organizaciones de migrantes preocupadas por desconocimiento de candidatos $(24 / 02 / 2019)$

2. Las ofertas de los candidatos a la Alcaldía de Quito no incluyen plan presupuestario $(10 / 03 / 2019)$

Como se había explicado anteriormente, la Democracia Participativa se relaciona con la validación de los mecanismos para que la gente decida sobre el municipio. Por lo tanto, el énfasis en esta cuestión de Yunda y Maldonado viene dado porque es un tema transversal, pero, además, es uno de las cuestiones que poseen gracias a su trascendencia. Por ello, "enfatizan desproporcionadamente los asuntos que "poseen" (Dolezal, 2014, p. 57). 
Figura 6. Resultados de temática: Democracia Participativa

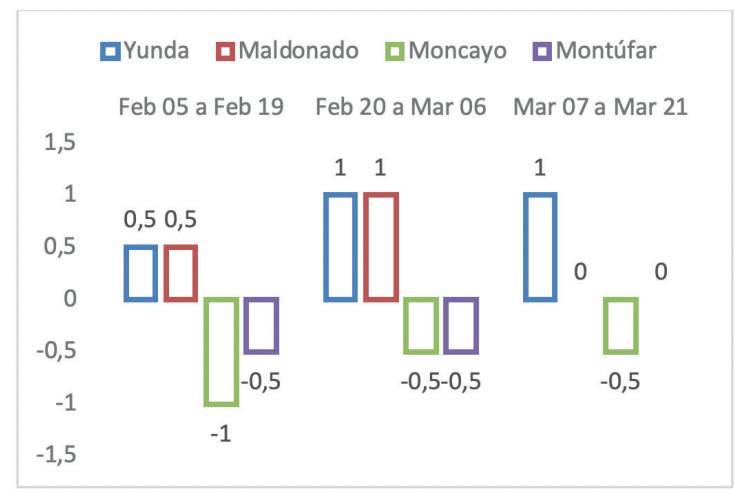

Fuente: Elaborado por los autores.

Finalmente, en la temática de Inclusión Social la candidata que más intervino, frente a su plan de campaña, fue Luisa Maldonado (Figura 7), que hizo de este tema una de sus banderas de campaña. Los tres candidatos restantes no se manifestaron en la misma medida que en sus planes de campaña, dejando de lado este apartado en función de relacionarse con otros temas. Por lo tanto, resalta el silencio de los candidatos en temas sobre la migración, discapacidad y precarización laboral, temas que deberían regir la agenda política por ser de las mayores inquietudes ciudadanas, de acuerdo con El Comercio (14/02/2019).

En los medios aparecieron informaciones relacionadas con la definición del Código de la Democracia que en algunos articulados dejan al libre albedrío de los movimientos y partidos políticos, el tema de la paridad e inclusión. Las noticias más importantes de esta sección fueron las siguientes, a más de las antes mencionadas:

1. El CNE resolvió invitar a la OEA para las elecciones seccionales (11/02/2019)

2. Elecciones recuerdan necesidad de reformas (17/02/2019)

3. 504 medios se monitorean en la campaña electoral (28/02/2019)

Este resultado es muy ilustrativo con respecto a lo que Dolezal et al. (2014) advierten: se nota cómo Maldonado enfatiza el tema de inclusión —una cuestión que le pertenece fuertemente y de la que habla reiteradamente en su agenda política-, mientras que los otros candidatos prefieren no confrontarse directamente con ella porque saben que no es una cuestión de su propiedad. Tiene sentido, dado que, como manifiesta Petrocick (1996), las campañas electorales tienen la función de convencer a los electores. Por ello, no es beneficioso apropiarse de una cuestión que le pertenece popularmente a otro candidato. 
Figura 7. Resultados de temática: Inclusión social

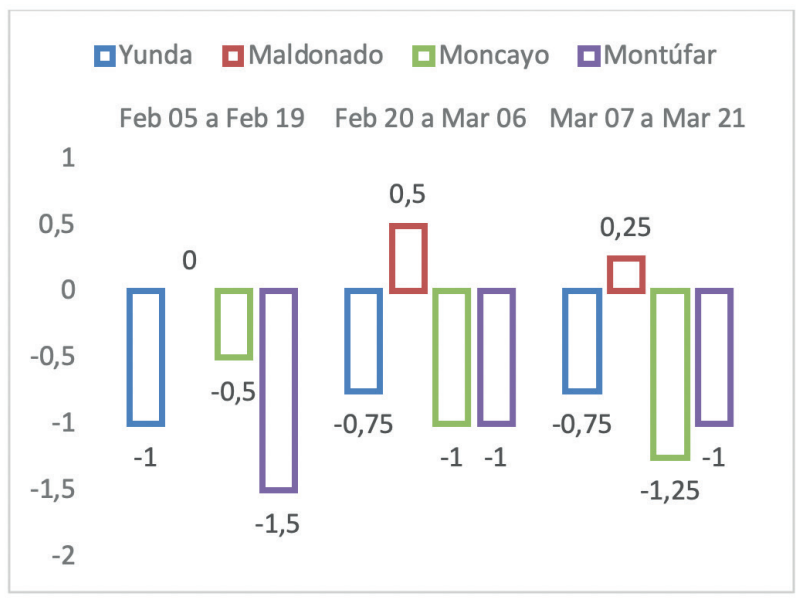

Fuente: Elaborado por los autores.

\section{DISCUSIÓN}

Las redes sociales digitales han transformado la manera de gestionar campañas políticas. En una democracia "imperfecta" como la ecuatoriana, Twitter ha sido acogido como una opción para acercar los ciudadanos/votantes a sus candidatos. Esto, a la luz de las teorías de la propiedad y de la prominencia de las cuestiones, esboza dos conclusiones: por un lado, el valor de la capacidad de los candidatos (Petrocick, 1996 y D’Ancona, 2016) y, por el otro, la capacidad que tienen los candidatos de responder rápidamente a las exigencias de los eventos que se presentan en la agenda mediática.

Es importante señalar la horizontalidad que implica el uso de redes sociales, pues activa la potencialidad de los electores para interpelar constantemente las propuestas de los candidatos. Ante esta realidad, los candidatos responden de dos maneras; en primer lugar, los datos de esta investigación confirman que hay una multiplicidad de voces al momento de presentar y enmarcar las propuestas de los candidatos, ellos demuestran a través de fotos y etiquetas a sus posibles aliados.

En segundo lugar, desde un inicio los candidatos buscan la aprobación ciudadana a través de la creación de planes de trabajo relacionados a temas presentes en las demandas y necesidades de los electores, como la lucha contra la corrupción. Esto implica que desde un principio los partidos generen narrativas apuntando a un rival (como en el caso de Luisa Maldonado contra Paco Moncayo) o bien despejar las dudas permanentes acerca de la lucidez de su posible gestión. Esto está en concordancia con el análisis de Budge and Farlie (1983), quienes notaron que los candidatos típicamente enfatizan un set común de cuestiones incluso cuando su credibilidad, en cada una, varíe. 
La capacidad que tienen los candidatos de responder rápidamente a las exigencias de la agenda mediática se relaciona con la teoría de la prominencia de las cuestiones. Si bien los candidatos han demostrado que las propuestas están basadas en las preocupaciones de los quiteños: desempleo, crisis económica, inseguridad, delincuencia, pésimo servicio de transporte público, pobreza y corrupción, se mira cómo los políticos también utilizan los mecanismos de priming y framing a su conveniencia.

Existe una afectación del tema político correspondiente a la incidencia directa de la agenda mediática sobre ciertas cuestiones que cada candidato adoptó como su fuerte, como el tema principal de su campaña electoral. Estas decisiones políticas se establecen al sentir más dominio en una temática particular y, a su vez, denotar la debilidad de sus oponentes. Podemos ver como las temáticas de propuesta electoral y los temas abordados por los medios impresos y digitales "El Comercio, La Hora y Últimas Noticias" buscan cercanía con lo que los públicos quieren ver, leer o escuchar.

En lo relacionado a los hallazgos, es importante señalar que dos temáticas no encontraron mayor eco en las publicaciones de los candidatos: Inclusión Social y Seguridad. En el primero, se puede esbozar una conclusión a partir de la única candidata con saldo positivo, Luisa Maldonado. Uno de sus pilares comunicacionales fue mostrarse como una candidata mujer y con discapacidad física. A partir de ello, la candidata construyó un discurso empático hacia la problemática, y lo sostuvo en sus intervenciones digitales, además demarcándose de sus opositores, que apenas lo mencionaron, en sus planes y en sus tuits. Por otro lado, el tema de Seguridad fue otra temática apenas mencionada por tres candidatos, salvo Paco Moncayo, que también a partir de su perfil profesional y su pasada gestión, convirtió a esta problemática en la oportunidad de sobresalir, además de manera constante durante los 45 días de campaña electoral.

Uno de los hallazgos que también vale la pena comentar es el comportamiento de César Montúfar, que en todos los seis grandes temas -revisados en este análisis- presenta números negativos, es decir se refirió de manera menos activa que lo que hizo en su plan. Con mirada en los datos globales, el candidato utilizó cerca de su 30\% de sus intervenciones en hablar de sus encuentros electorales y demás actividades proselitistas, lo que indicaría que dejó marcada una línea de pensamiento en su plan, pero utilizó Twitter como un canal de campaña y no de profundización de los temas y su visión.

Finalmente, es necesario referirse al comportamiento de Jorge Yunda, ganador de los comicios; el candidato que más usó Twitter durante la campaña electoral (1117 tuits en total). Como se mencionó, Yunda utilizó su cuenta en un $70 \%$ para hablar de eventos proselitistas y la temática Ecología, medio ambiente y fauna urbana. En las temáticas analizadas tuvo alguna participación positiva en Democracia Participativa, Corrupción y Productividad, pero nada destacado. Es interesante mirar que este candidato hizo menos caso a la agenda mediática para determinar su discurso, él siguió con los temas que lo posicionaron durante años como un animalista y un animador. Interesante, pues este trabajo contribuyó, de alguna manera que será interesante revisar en futuras investigaciones, en su victoria. 


\section{BIBLIOGRAFÍA}

ARAGONÉS, E. y MICAEL-CASTANHEIRA, M. (2012). "Electoral competition through issue selection" en Barcelona GSE Working Paper Series, 641, pp. 1-32.

BÉLANGER, E. y MEGUID, B. (2008). 'Issue Salience, Issue Ownership, and Issue-based Vote Choice’ en Electoral Studies, 27 (3), pp. 477-91. https://doi.org/10.1016/j. electstud.2008.01.001

BUDGE, I. (1982). "Electoral volatility: Issue effects and basic change in 23 post-war democracies" en Electoral Studies, 1, pp. 147-168. https://doi.org/10.1016/02613794(82)90001-4

BUDGE, I. y FARLIE, D. (1983). "Party competition - selective emphasis or direct confrontation? An alternative view with data”. En H. Daalder \& P. Mair (Eds). Western European party systems. Londres: Sage.

CENTENO, M.A. y LAJOUS, A. (2017). The Age of Perplexity: Rethinking the World we Knew. Madrid: Fundación BBVA, OpenMind, Penguin Random House Grupo Editorial.

CHAFFEE, S. y WILSON, D. (1977). "Media Rich, Media Poor: Two Studies of Diversity in Agenda Holding" en Journalism Quarterly, pp. 466-476. https://doi. org/10.1177/107769907705400304

CHONG, D. y DRUCKMAN, J. N. (2007). "Framing theory” en Annu. Rev. Polit. Sci., 10, pp.103-126. https://doi.org/10.1146/annurev.polisci.10.072805.103054

COHEN, B. (1963). The Press and Foreign Policy. Princeton: Princeton University Press.

CONSEJO NACIONAL ELECTORAL (2019). Resultados 2019. Recuperado el 25 de agosto de 2019. Disponible en https://resultados2019.cne.gob.ec

DOMÍNGUEZ, E. (2012). Medios de comunicación masiva. México. Red Tercer Milenio S.C.

DOLEZAL, M., ENNSER-JEDENASTIK, L., MÜLLER, W.C. y WINKLER, A. K. (2014). "How parties compete for votes: A test of saliency theory" en European Journal of Political Research ,53, pp. 57-76. https://doi.org/10.1111/1475-6765.12017

GENE-ZUCKER, (1978). "The Variable Nature of News Media Influence" en Annals of the International Communication Association, 2 (1), pp. 225-240. https://doi.org/10.1 080/23808985.1978.11923728

GREEN, J. y JENNINGS, W. (2012). 'The Dynamics of Issue Competence and Vote for Parties in and Out of Power: An Analysis of Valence in Britain, 1979-1997' en European Journal of Political Research, 51(4), pp. 469-503. https://doi.org/10.1111/j.14756765.2011.02004.x

IYENGAR, S., PETERS, M.D. y R. KINDER, D. R. (1982) “Experimental Demonstration of the 'Not-So-Minimal' Consequences of Television News Programs." En American Political Science Review ,76, pp. 848-58. https://doi.org/10.1017/S000305540018966X

JENSEN, K. B. (2014). La comunicación y los medios: metodologías de investigación cualitativa y cuantitativa. Fondo de Cultura Económica.

JEWITT, C. (2005). Multimodality, "reading", and "writing" for the 21 st century. Discourse: studies in the cultural politics of education, 26(3), pp. 315-331. https://doi. org/10.1080/01596300500200011

KIOUSIS, S., STRÖMBÄCK, J., y MCDEVITT, M. (2015). "Influence of issue 
decision salience on vote choice: Linking agenda setting, priming, and issue ownership" en International Journal of Communication, 9, 22.

KLEINNIJENHUIS, J., VAN ATTEVELDT, W. y DEKKERS, V. (2018). "Partial priming: how issue news shapes issue saliency, which shapes turnout but not the vote" en Acta Politica, 53 (4), pp. 569-589. https://doi.org/10.1057/s41269-018-0104-2

LACHAT, R. (2014). "Issue Ownership and the Vote: The Effects of Associative and Competence Ownership on Issue Voting” en Swiss Political Science Review, 20 (4), pp. 727-40. https://doi.org/10.1111/spsr.12121

LANG, G. E. y LANG, K. (1981). "Mass communication and public opinion: strategies for research". En M. Rosenberg y R. H. Turner (Eds.). Social Psychology: Sociological Perspectives. Nueva York. Basic Books.

LATINOBARÓMETRO (2018). Informe 2018. Corporación Latinobarómetro. Disponible en: http://www.latinobarometro.org/latdocs/INFORME_2018_LATINOBAROMETRO.pdf $+\& \mathrm{~cd}=1 \& \mathrm{hl}=\mathrm{es} \& \mathrm{ct}=\mathrm{clnk} \& \mathrm{gl}=\mathrm{ec}$

MARKS, G. (Ed.), (2007). "Special symposium: Comparing measures of party positioning: expert, manifesto, and survey data" en Electoral Studies, 26(1), pp. 1-141. https:// doi.org/10.1016/j.electstud.2006.03.009

MCCOMBS, M., y SHAW, D. (1972). "The Agenda-Setting Function of Mass Media" en The Public Opinion Quarterly, 36, 2, (Summer, 1972), pp. 176-187. https://doi. org/10.1086/267990

ORTELLS-BADENES, S. (2014). "Los criterios de noticiabilidad periodística en los programas de infoentretenimiento" en Textual \& Visual Media, (7), pp. 207-220.

PETROCIK, J (1996). "Issue Ownership in Presidential Elections, with a 1980 Case Study" en American Journal of Political Science, 40 (3), pp. 825-850. https://doi. org/10.2307/2111797

PETRONE, F. (2009). Estudio de agenda setting: Conceptos, metodología y abordajes posibles. Universidad de Buenos Aires, Buenos Aires.

QUITO: LA GUÍA ELECTORAL. (2019). En PlanV. Recuperado el 25 de agosto de 2019 de https:/www.planv.com.ec/historias/politica/quito-la-guia-electoral-elegir-al-nuevo-alcalde-metropolitano

ROSKOS-EWOLDSEN, D. R., ROSKOS-EWOLDSEN, B., \& CARPENTIER, F. R. D. (2002). "Media priming: A synthesis" en Media effects , pp. 107-130 Routledge.

SCHEUFELE, D. A. (1999). "Framing as a theory of media effects" en Journal of communication, 49(1), pp. 103-122. https://doi.org/10.1111/j.1460-2466.1999.tb02784.x

TRECHSEL, A. y MAIR, P. (2011). "When Parties (Also) Position Themselves: An Introduction to the EU Profiler" en Journal of Information Technology \& Politics, 8 (1), pp. 1-20. https://doi.org/10.1080/19331681.2011.533533

WALGRAVE, S., TRESC, A. y LEFEVERE, J. (2015). “The Conceptualisation and Measurement of Issue Ownership" en West European Politics, 38 (4), pp. 778-796. https:// doi.org/10.1080/01402382.2015.1039381

WALGRAVE, S., LEFEVERE, J. y TRESCH, A. (2012). “The Associative Dimension of Issue Ownership" en Public Opinion Quarterly, 76 (4), pp. 771-82. https://doi. org $/ 10.1093 /$ poq $/$ nfs023

ZHU, J., y BLOOD, D., (1996). "Media Agenda-Setting Theory Review of a 25 Year Research Tradition” en Perspectives, 8, pp. 97-149. 